\title{
Improved Mass Accuracy for Higher Mass Peptides by Using SWIFT Excitation for MALDI-FTICR Mass Spectrometry
}

\author{
Li Jing, ${ }^{\mathrm{a}, *}$ Chunyan Li, ${ }^{\mathrm{a}, *}$ Richard L. Wong, ${ }^{\mathrm{b}}$ Desmond A. Kaplan, ${ }^{\mathrm{c}}$ and \\ I. Jonathan Amster ${ }^{\mathrm{a}}$ \\ ${ }^{a}$ Department of Chemistry, University of Georgia, Athens, Georgia, USA \\ ${ }^{\mathrm{b}}$ Bristol-Myers Squibb, Hopewell, New Jersey, USA \\ ${ }^{\mathrm{c}}$ Bruker Daltonics, Billerica, Massachusetts, USA
}

\begin{abstract}
Stepwise-external calibration has previously been shown to produce sub part-per-million (ppm) mass accuracy for the MALDI-FTICR/MS analyses of peptides up to $\mathrm{m} / z 2500$. The present work extends these results to ions up to $\mathrm{m} / \mathrm{z} 4000$. Mass measurement errors for ions of higher mass-to-charge are larger than for ions below $\mathrm{m} / \mathrm{z} 2500$ when using conventional chirp excitation to detect ions. Mass accuracy obtained by using stored waveform inverse Fourier transform (SWIFT) excitation was evaluated and compared with chirp excitation. Analysis of measurement errors reveals that SWIFT excitation provides smaller deviations from the calibration equation and better mass accuracy than chirp excitation for a wide mass range and for widely varying ion populations. (J Am Soc Mass Spectrom 2008, 19, 76-81) (c 2008 American Society for Mass Spectrometry
\end{abstract}

$\mathrm{A}$ ccurate mass measurement has long been recognized as a powerful tool in mass spectrometry, enabling the assignment of unique elemental compositions for small molecules (MW < $500 \mathrm{Da})$ [1], and more recently, used for making higher confidence peptide identifications [2]. Accurate mass measurements are carried out using a variety of mass spectrometers. Time-of-flight (TOF) mass spectrometers now provide accuracy within $10 \mathrm{ppm}[3,4]$. Orbitrap mass measurement accuracies have been reported to be 2 to 5 ppm [5, 6]. Fourier-transform ion cyclotron resonance (FTICR) mass spectrometry, developed by Comisarow and Marshall [7,8], currently provides the best mass resolution and mass accuracy $(<1 \mathrm{ppm})$ of all types of mass analyzers [9-11] and has proven to be useful for protein identification by database searching $[2,12]$. Mass measurement accuracy (MMA) at the sub part-per-million (ppm) level using internal calibration $[13,14]$ and several ppm using external calibration have been demonstrated $[15,16]$, and these have led to much greater identification specificity, as described in recent reviews [17, 18].

For FTICR/MS, space-charge is the principal cause of mass measurement error [15, 19, 20]. The best MMA is obtained by using internal calibration, as this eliminates global space-charge effects [16]. Conventionally, internal calibration is achieved by mixing a calibrant

Address reprint requests to Dr. J. Amster, Department of Chemistry, University of Georgia, Athens, Georgia 30602, USA. E-mail: jamster@ uga.edu

* These two authors contributed equally to this work. with the analyte. Internal calibration can be achieved without adding calibrant directly into the analyte by using a dual-spray source $[14,21]$ in ESI experiments or by using the internal calibration on adjacent samples (InCAS) calibration method [22, 23] in MALDI experiments. However, internal calibration requires having both calibrant and analyte ions present at the same time in the analyzer cell, which congests the mass spectrum and can lead to overlapping peaks. Such issues can be avoided with external calibration, but space-charge shifts of cyclotron frequencies can lead to systematic errors in mass measurement. The most accurate external calibration procedures rely on a calibration equation that accounts for ion intensities [15, 16, 24], or for matching the ion abundance between the analyte and calibrant spectra, e.g., by automatic gain control (AGC) $[14,25]$. However, AGC is not applicable to MALDIFTICR measurements due to the large shot-to-shot variation in ion intensity that is characteristic of MALDI.

We recently described a two-step external calibration procedure for MALDI-FTICR stepwise-external calibration [26], in which a mass spectrum is first acquired at low trapping potential, with sub-ppm mass accuracy by external calibration. This is then followed by reacquiring the spectrum at higher trapping potential for the same sample, which provides higher dynamic range. The peaks from the low trapping potential spectrum are used as "confidently-known masses" or pseudocalibrants for internal calibration of the spectrum collected at higher trapping potential. Stepwise-external calibration provides many advantages of internal cali- 
bration without its disadvantages. Mass accuracy has been improved two to four times for ions below massto-charge ratio $(\mathrm{m} / \mathrm{z})$ 2500, and a root-mean-square (RMS) error of 0.9 ppm has been demonstrated for 609 measured peptide ions, whose mass errors distribute in a Gaussian fashion. Although most tryptic peptides have molecular weights less than $2500 \mathrm{Da}$, higher mass peptides have greater information content, and the protein identification rate can be increased significantly by incorporating data from spectra acquired with tuning parameter to enhance ions of higher mass-to-charge [27]. When the stepwise-external calibration approach is applied to ions above $m / z 2500$, we find that the RMS error increases to $\sim 3 \mathrm{ppm}$ [27]. Work by Masselon et al. suggests that random error in FTICR mass measurement may be related to the type of excitation waveform used for ion detection [24]. In our previous work, all measurements on the Bruker FTICR mass spectrometer were made using frequency-sweep (chirp) excitation. Smith and coworkers have shown that data collected using stored-waveform inverse Fourier transform (SWIFT) excitation provides better MMA than using chirp excitation for ESI measurements of ions up to $\mathrm{m} / \mathrm{z}$ 1800 [24]. Presumably, this is a result of a more uniform power applied across all frequencies leading to a more uniform distribution of radii of gyration for all ions by SWIFT compared with chirp [28]. These results have encouraged us to examine this approach to improving mass accuracy for higher mass singly-charged ions when using stepwise-external calibration.

Here we present results of a comparison of chirp and SWIFT excitation for accurate mass measurement in MALDI-FTICR/MS using stepwise-external calibration. First, we evaluate the standard deviation (SD) of internal calibration mass error as a function of ion excitation power using chirp and SWIFT excitation. We also examine the MMA that can be obtained for ions up to $\mathrm{m} / \mathrm{z} 4000$ by chirp and SWIFT excitation using two calibration procedures, conventional external calibration [19] and stepwise-external calibration [26]. We show that SWIFT excitation provides significantly better mass accuracy, particularly at higher mass-tocharge, than can be achieved by chirp excitation when using stepwise-external calibration.

\section{Experimental}

Bovine serum albumin (BSA) and chicken egg albumin (ovalbumin) were purchased from Sigma (St. Louis, $\mathrm{MO})$. Each protein sample was dissolved in alkaline solution (10 $\mathrm{mM}$ ammonium bicarbonate) to make a 1 $\mathrm{mg} / \mathrm{mL}$ solution and denatured by heating at $90{ }^{\circ} \mathrm{C}$ for 10 min. Disulfide bonds were reduced with tris (2carboxythyl) phosphine (Pierce Biotechnology, Rockford, IL). Denatured proteins were digested overnight at $37^{\circ} \mathrm{C}$ using trypsin (Promega, Madison, WI) at a 1:50 protease/protein ratio (by mass); $400 \mathrm{~nL}$ of the digested proteins was applied to a stainless steel MALDI target and $400 \mathrm{~nL}$ of 1 M 2,5-dihydroxybenzoic acid (DHB)
(Lancaster, Pelham, NH) in acetonitrile/water/TFA (50\%:50\%:0.1\%, vol/vol/vol) was added as the MALDI matrix.

Mass spectra were collected using a BioApex 7 tesla FTICR mass spectrometer equipped with an intermediate pressure Scout 100 MALDI source (Bruker Daltonics Inc, Billerica, MA). Ions from up to 10 laser desorption events were accumulated in the source hexapole ion guide, and then the ions were released from the ion guide and transmitted to the analyzer cell with electrostatic ion optics. The delay (D2) between ion extraction from the source hexapole and the gating of the cell entrance electrode potentials, EV1 $(-7.0 \mathrm{~V})$ and EV2 $(-1.5 \mathrm{~V})$, back to the trapping potential was set to 4.0 $\mathrm{ms}$ to enhance the detection of heavier ions [27]. No sidekick deflection was employed (DEV2 $=0)$. The ions were excited by using either a chirp waveform or a SWIFT waveform [29]. The chirp excitation consisted of 95 frequencies with a dwell time of $20 \mu$ s per step, and frequency increment of $2000 \mathrm{~Hz}$, yielding a $1.9 \mathrm{~ms}$ signal duration that covered the frequency range of 21,511 to $215,277 \mathrm{~Hz}$, corresponding to $m / z 5000$ to 500 . The optimal excitation voltage was $100 \mathrm{~V}_{p-p}$ amplitude. For SWIFT excitation experiments, waveforms were produced by a PXI box with a PXI-8184 embedded controller and a PXI-5412 arbitrary waveform generator (National Instruments, Austin, TX). The power spectrum was designed to have flat amplitude over the frequency range of 21,511 to $215,277 \mathrm{~Hz}$ (corresponding to $m / z$ 500-5000). A quadratic phase versus frequency function was used for the inverse Fourier transform calculation to yield a SWIFT excitation waveform that had relatively constant amplitude versus time [29, 30]. The excitation waveform was digitized to 32,768 points, which were read out at rate of $16,667,000$ points/s to yield an excitation signal with a duration of $1.966 \mathrm{~ms}$. After ion excitation, a $512 \mathrm{~K}$ point transient was acquired, apodized with a sinebell function, and padded with one zero-fill before fast Fourier transformation and magnitude calculation. Only monoisotopic peaks with a signal-to-noise ratio $(\mathrm{S} / \mathrm{N})>3$ were used to study the mass accuracy.

For stepwise-external calibration, the first mass spectrum is acquired at a low trapping potential $(0.60 \mathrm{~V})$ using external calibration, eq 1, where the average mass accuracy is quite good (RMS error $<0.5 \mathrm{ppm}$ ). The second mass spectrum is acquired for the same sample at higher trapping potential $(1.10 \mathrm{~V})$ to increase the detection dynamic range by a factor of 5 to 10 [26]. For peaks that appear in both mass spectra, the fairly accurate mass values from the first mass spectrum are used to calibrate the masses in the higher trapping potential spectrum using eq 2. Multi-linear regression is used to obtain the calibration constants by fitting the measured frequencies and intensities to the masses that are determined from the first mass spectrum. All statistical data were analyzed using Microsoft Excel. Multilinear regression to obtain the constants for the calibration equations was performed using software devel- 
oped in our laboratory (available for download at http:/ / amstersgi.chem.uga.edu/html/software.html).

$$
\begin{aligned}
& \frac{m}{z}=\frac{A}{f+B} \\
& \left(\frac{m}{z}\right)_{i}=\frac{A}{f_{i}+B+C \cdot I_{i}}
\end{aligned}
$$

\section{Results and Discussion}

\section{Error Analysis for Calibrant Points}

Experiments were first performed to compare the standard deviations of mass errors that result from using chirp and SWIFT excitation waveforms with internal calibration. Five mass spectra of the tryptic peptides of BSA were acquired for chirp or SWIFT excitation at different values of excitation amplitude, normalized to a value of $100 \%$ for the power required to eject all ions. In this manner, we were able to compare the chirp and SWIFT excitation of ions to a similar cyclotron radius. From each spectrum, eight monoisotopic peaks were selected and fit by linear regression to eq 1 , where $f$ is the measured frequency of the ions, $m / z$ is the theoretical mass-to-charge value, and $A$ and $B$ are constants that are related to the magnetic field strength and radial components of trapping potential and the global space charge field, respectively [19].

Figure 1 shows the deviation between measurements of the eight masses used for calibration and the values calculated from eq 1, plotted against normalized ion excitation power for chirp and SWIFT excitation; the height of the error bars represent 1 SD based on five replicate measurements at each power value. As can be seen from this plot, the SD of the mass measurement error for both excitation methods depends on the ion

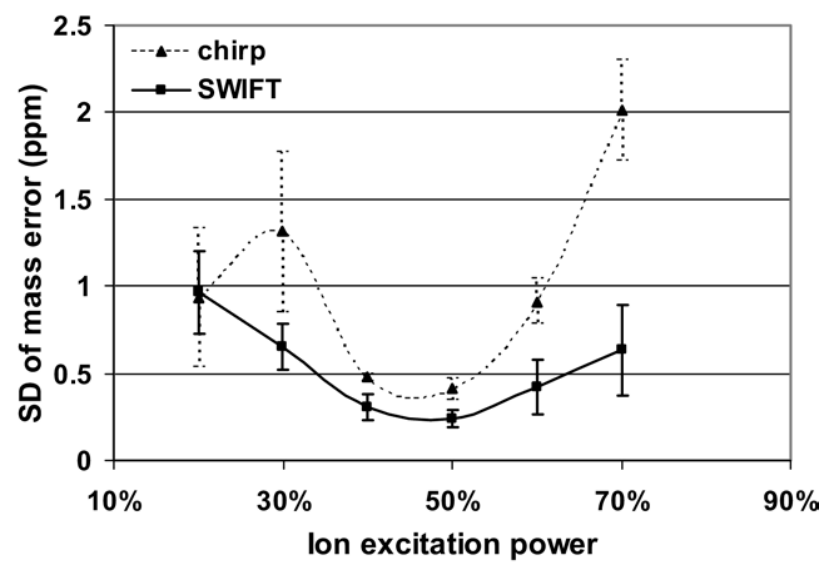

Figure 1. Standard deviation of calibration errors obtained from the linear fit to eq $1 \mathrm{vs}$. ion excitation power (the power to eject all ions is normalized to $100 \%$ ) using chirp or SWIFT excitation. Five measurements were averaged at each condition and the error bar representing $1 \mathrm{SD}$ of the mean. excitation power and the smallest errors are achieved when ion excitation power is optimized $(\sim 50 \%)$. When the ion excitation power is either larger or smaller than the optimal value, the deviation from the calibration equation becomes larger. The curve for data collected using chirp displays an irregular trend when the ion excitation power is $20 \%$ of the value required for ejection, which results from the peaks at high mass region falling below the minimum $\mathrm{S} / \mathrm{N}(<3)$ and being excluded as calibrants. SWIFT excitation is found to provide a smaller SD than chirp at all excitation powers and maintains an acceptably small value across a much wider range of excitation values. This shows that mass accuracy is less sensitive to tuning of the excitation power for SWIFT versus chirp measurements. While the data shown in Figure 1 was collected at low trapping potential $(0.6 \mathrm{~V})$, the same results are observed at the higher trapping potential used for stepwise calibration (1.1 V).

The total ion intensity is also compared for the same dataset using chirp and SWIFT. They are essentially the same when the same ion excitation power is used and the highest total ion intensity is achieved when the ion excitation power is around 50\% (data not shown). These results indicate that accurate mass measurements can be obtained under conditions of optimal sensitivity via both chirp and SWIFT. SWIFT excitation provides better mass accuracy than chirp and provides good mass accuracy even when the ion excitation power is not tuned to its optimal value.

\section{Stepwise-External Calibration for Ions up to $\mathrm{m} / \mathrm{z}$ 4000}

Stepwise-external calibration was examined for higher mass tryptic peptides, using an ovalbumin tryptic digest. Thirty mass spectra were collected at 0.60 and 1.10 $\mathrm{V}$ cell trapping potential using either chirp or SWIFT excitation with $50 \%$ ion excitation power, yielding a total of 120 spectra. By using a low trapping potential $(0.60 \mathrm{~V}$ for this experiment), the space charge-induced frequency shifts are significantly reduced due to the smaller ion capacity of the analyzer cell, so that highly accurate mass values can be obtained using external calibration [26]. However, the lower ion capacity of the analyzer cell reduces the $\mathrm{S} / \mathrm{N}$ and dynamic range of the mass spectra obtained in this manner [26]. The mass spectra acquired at higher trapping potential $(1.10 \mathrm{~V})$ are used to recover the dynamic range lost in the lower trapping potential experiment. Stepwise-external calibration is achieved by using the mass values measured at low trapping potential as calibration reference masses for the spectrum acquired at higher trapping potential via eq 2, where $I_{i}$ is the peak intensity of a particular ion, $(\mathrm{m} / \mathrm{z})_{\mathrm{i}}$. Parameter $C$ acts as a correction factor for local space-charge effects, i.e., the different interaction between ions of the same mass-to-charge versus ions of different mass-to-charge [24]. The cali- 
(a) Chirp, external calibration

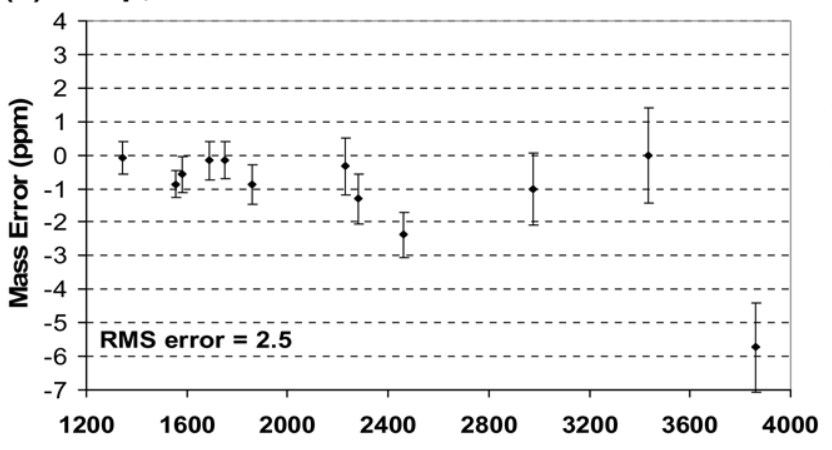

(b) Chirp, stepwise calibration

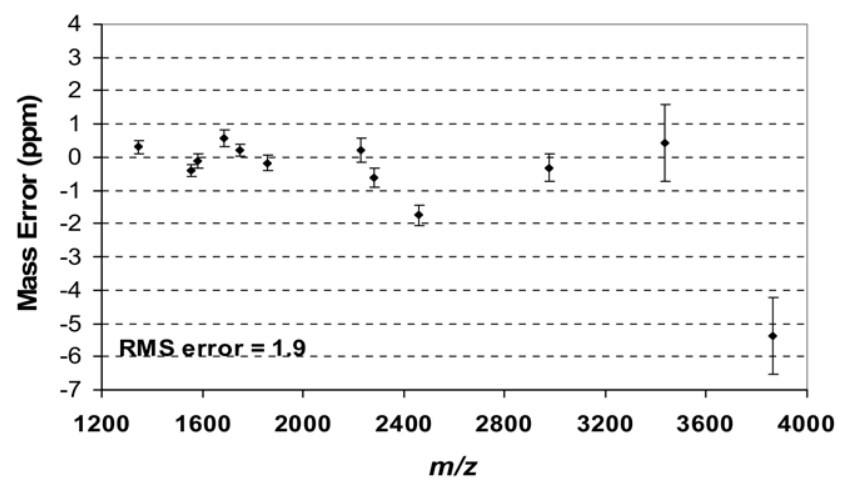

(c) SWIFT, external calibration

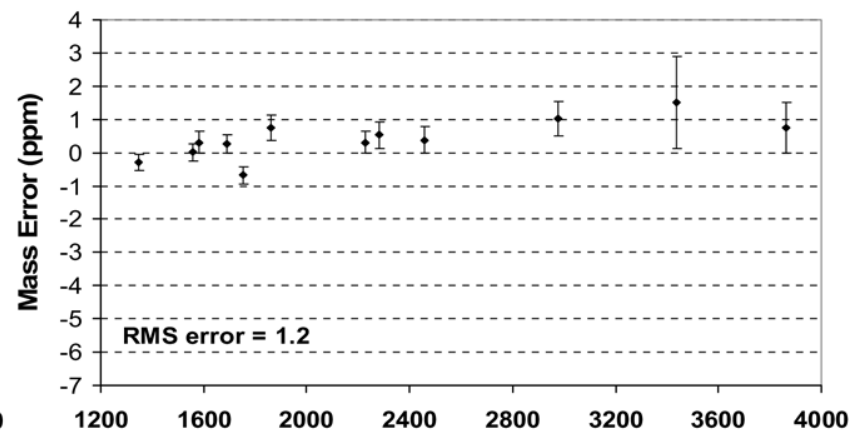

(d) SWIFT, stepwise calibration

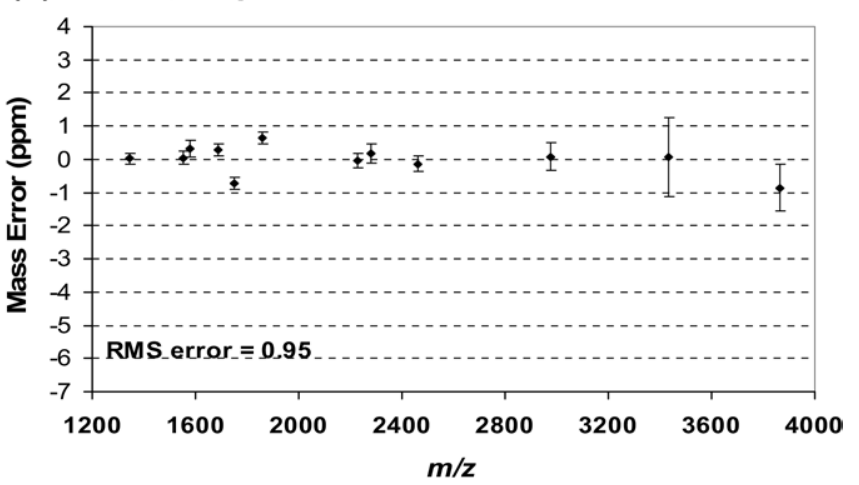

Figure 2. Average calibration errors obtained from external calibration (eq 1) and stepwise-external calibration (eq 2), with the error bar being 1 SD for 30 replicates. (a) chirp for external calibration, (b) chirp for stepwise-external calibration, (c) SWIFT for external calibration, and (d) SWIFT for stepwise-external calibration.

bration coefficients $A, B$, and $C$ are independent of the index $i$. This calibration method takes into account both global and local space-charge effects and has been demonstrated to improve the mass accuracy when compared to the application of calibration eq 1 [26]. To calibrate a mass spectrum acquired at higher trapping potential using eq 2 , one need at least three reference values from the low potential mass spectrum. The corresponding peaks are identified in the high potential mass spectrum, and their frequencies, $\mathrm{m} / \mathrm{z}$ values, and intensities are used to obtain the calibration constants for eq 2. To get the best overall fit for multi-linear regression, all isotopic peaks with $S / N>20$ were used to do the stepwise-external calibration and 12 monoisotopic peaks over a wide range (from $\mathrm{m} / \mathrm{z} 1346$ to $\mathrm{m} / \mathrm{z}$ 3863) were selected from each high potential spectrum to evaluate MMA.

Mass errors for each reference monoisotopic $\mathrm{m} / \mathrm{z}$ value obtained for the external and stepwise-external calibration are plotted in Figure 2. Figure 2a and c show the mass errors obtained at high trapping potential (1.10 V) for standard external calibration using eq 1 with chirp and SWIFT excitation, respectively. Figure $2 \mathrm{~b}$ and $\mathrm{d}$ show the mass errors obtained for the same datasets but calibrated with stepwise-external calibration using eq 2 with chirp and SWIFT excitation, respectively. The height of the error bars indicates 1 SD for 30 spectra obtained under the same conditions. As seen in Figure 2 , the mass errors are noticeably reduced when stepwise-external calibration is applied in data collected from both chirp and SWIFT. As expected, the mass error (RMS error $=2.5 \mathrm{ppm}$ ) is the largest with external calibration using chirp excitation waveform (Figure 2a) and SWIFT-excite data calibrated with stepwise-external calibration yields the smallest mass error (RMS error $=0.95 \mathrm{ppm}$ ) (Figure 2d). In addition, the mass errors for ions with $\mathrm{m} / \mathrm{z}>2000$ indicated that better mass accuracy is achieved by using SWIFT, for both external calibration and stepwise-external calibration (Figure 2c and d).

Table 1 compares the SD and RMS of mass errors that were calculated for 30 chirp-excite spectra and 30 SWIFT-excite spectra obtained at high trapping potential with external calibration, internal calibration and stepwise-external calibration. Three different mass ranges were considered for data from each excitation waveform and all errors are expressed in ppm. The SD and RMS of mass errors from external calibration are noticeably reduced when internal calibration or stepwise-external calibration is used, and the errors for stepwise-external calibration results are only slightly larger than by internal calibration. As previously observed [26], by using stepwise-external calibration, the values of RMS and SD are around $0.9 \mathrm{ppm}$ for chirp excitation of ions less than $\mathrm{m} / \mathrm{z} 2500$, a $\sim$ twofold im- 
Table 1. Error analysis for data collected using chirp and SWIFT excitation with external calibration, internal calibration, and stepwise-external calibration

\begin{tabular}{|c|c|c|c|c|c|c|}
\hline & \multicolumn{4}{|c|}{$\begin{array}{c}2500<m / z \\
<4000\end{array}$} & \multicolumn{2}{|c|}{ All ions } \\
\hline & SD & RMS & SD & RMS & SD & RMS \\
\hline Chirp external & 1.4 & 1.6 & 3.6 & 4.2 & 2.2 & 2.5 \\
\hline Chirp internal & 0.54 & 0.55 & 2.7 & 2.8 & 1.4 & 1.4 \\
\hline Chirp stepwise & 0.83 & 0.85 & 3.2 & 3.6 & 1.9 & 1.9 \\
\hline SWIFT external & 0.74 & 0.78 & 1.9 & 2.1 & 1.2 & 1.2 \\
\hline SWIFT internal & 0.45 & 0.46 & 1.2 & 1.2 & 0.71 & 0.71 \\
\hline SWIFT stepwise & 0.55 & 0.56 & 1.7 & 1.7 & 0.95 & 0.95 \\
\hline
\end{tabular}

provement in mass accuracy compared with external calibration. For ions in the high mass range $(2500<\mathrm{m} / \mathrm{z}$ $<4000$ ), stepwise-external calibration with chirp excitation produces are relatively small improvement in MMA (RMS 3.6 ppm versus $4.2 \mathrm{ppm}$ for standard external calibration). However, even internal calibration produces large errors at these higher masses when using chirp excitation (RMS 2.8 ppm). In contrast, for SWIFT excitation, internal calibration produces RMS errors of only $1.2 \mathrm{ppm}$. Stepwise-external calibration with SWIFT excitation produces RMS error values of $0.56,1.7$, and $0.95 \mathrm{ppm}$ for the low mass range, high mass range, and the entire mass range, respectively. For the same mass range and the same calibration method, the values of SD and RMS from chirp-excite experiments are almost double those obtained using SWIFT. Thus, it can be confidently stated that mass errors are reduced by using SWIFT excitation, particularly for ions of higher mass-to-charge. Through the comparison of these waveforms and calibration procedures, we are able to improve MMA to sub-ppm for ions up to $\mathrm{m} / \mathrm{z}$ 3000 , and to less than $2 \mathrm{ppm}$ for ions up to $\mathrm{m} / \mathrm{z} 4000$ by using SWIFT excitation and the stepwise-external calibration method.

These results suggest substantial advantages of this methodology for proteomics analysis by MALDIFTICR/MS. Batch tryptic digests of proteomes produce many thousands of peptides components, and even after offline liquid chromatography, individual fractions are highly complex. Such samples are highly amenable to the stepwise calibration procedure, as the larger number of peaks that appear in the mass spectrum acquired at low trapping potential allow a greater number of pseudo-calibrants for the stepwise calibration procedure, resulting in a more accurate calibration curve. Furthermore, the larger the number of components, the more uniform is the total ion intensity from fraction to fraction, which decreases space-charge frequency shifts that might otherwise limit mass accuracy in the first step of the stepwise calibration procedure, which relies on external calibration. Conversely, this procedure will have little utility for online-HPLC ESIFTICR/MS measurements, where the number of components present in a mass spectrum is relatively low, and the variation in total ion intensity is higher (in the absence of automatic gain control.)

\section{Conclusions}

The utilization of a SWIFT excitation waveform reduced mass errors significantly compared with chirp excitation, particularly for ions of higher mass-to-charge. SWIFT provides a flat power distribution across the range of $\mathrm{m} / \mathrm{z}$ values that are undergoing excitation, leading to more uniform cyclotron radii for all ions [28, 29]. Stepwise-external calibration yields a RMS error of $0.95 \mathrm{ppm}$ for ions up to $\mathrm{m} / \mathrm{z} 4000$ using SWIFT excitation. These data demonstrate that sub-ppm MMA can be achieved when SWIFT excitation is combined with stepwise-external calibration. This is particularly significant for MALDI measurements where there is considerate variation in ion intensity from spectrum to spectrum. We are currently working on extending these findings to proteomics measurements using MALDIFTICR/MS, as this level of MMA will allow us to perform searches at a mass tolerance of $3 \mathrm{ppm}$ (three times the RMS) with $99.7 \%$ confidence in the mass accuracy. It is noteworthy that these experiments were performed with a $7 \mathrm{~T}$ magnetic field; the MMA is expected to improve even further by using higher magnetic fields [31]. The improvement of MMA for ions up to $m / z 4000$ should benefit the analysis of complex mixtures such as batch proteolytic digests of protein mixtures, where higher dynamic range and higher mass accuracy is required.

\section{Acknowledgments}

The authors thank Dr. Paul Speir of Bruker Daltonics for providing the hardware and software necessary to perform SWIFT excitation. The authors are grateful for financial support from National Science Foundation (grant CHE-0316002) and National Institutes of Health (grant RR019767).

\section{References}

1. Gross, M. L. Accurate Masses for Structure Confirmation. I. Am. Soc. Mass Spectrom. 1994, 5, 57-57.

2. Conrads, T. P.; Anderson, G. A.; Veenstra, T. D.; Pasa-Tolic, L.; Smith, R. D. Utility of Accurate Mass Tags for Proteome-Wide Protein Identification. Anal. Chem. 2000, 72, 3349-3354.

3. Williams, J. D.; Flanagan, M.; Lopez, L.; Fischer, S.; Miller, L. A. D. Using Accurate Mass Electrospray Ionization Time-of-Flight Mass Spectrometry with In-Source Collision-Induced Dissociation to Sequence Peptide Mixtures. J. Chromatogr. A 2003, 1020, 11-26.

4. Wyatt, M. F.; Stein, B. K.; Brenton, A. G. Investigation into Accurate Mass Capability of Matrix-Assisted Laser Desorption/Ionization Timeof-Flight Mass Spectrometry, with Respect to Radical Ion Species. J. Am. Soc. Mass Spectrom. 2006, 17, 672-675.

5. Yates, J. R.; Cociorva, D.; Liao, L.; Zabrouskov, V. Performance of a Mass Analyzer with Orbital Trapping for Peptide Analysis. Anal. Chem. 2006, 78, 493-500.

6. Makarov, A.; Denisov, E.; Kholomeev, A.; Balschun, W.; Lange, O. Strupat, K.; Horning, S. Performance Evaluation of a Hybrid Linear Ion Trap/Orbitrap Mass Spectrometer. Anal. Chem. 2006, 78, 2113-2120.

7. Comisarow, M. B.; Marshall, A. G. Fourier Transform Ion Cyclotron Resonance Spectroscopy. Chem. Phys. Lett. 1974, 25, 282-283.

8. Comisarow, M. B.; Marshall, A. G. Frequency-Sweep Fourier Transform Ion Cyclotron Resonance Spectroscopy. Chem. Phys. Lett. 1974, 26 489-490.

9. Gorshkov, M. V.; Guan, S.; Marshall, A. G. Masses of Stable Neon Isotopes Determined at Parts per Billion Precision by Fourier Transform 
Ion Cyclotron Resonance Mass Spectrometry. Int. J. Mass Spectrom. Ion Processes 1993, 128, 47-60.

10. Rodgers, R. P.; White, F. M.; Hendrickson, C. L.; Marshall, A. G.; Andersen, K. V. Resolution, Elemental Composition, and Simultaneous Monitoring by Fourier Transform Ion Cyclotron Resonance Mass Spectrometry of Organosulfur Species Before and After Diesel Fuel Processing. Anal. Chem. 1998, 70, 4743-4750.

11. He, F.; Hendrickson, C. L.; Marshall, A. G. Baseline Mass Resolution of Peptide Isobars: A Record for Molecular Mass Resolution. Anal. Chem. 2001, 73, 647-650

12. Smith, R. D.; Anderson, G. A.; Lipton, M. S.; Pasa-Tolic, L.; Shen, Y.; Conrads, T. P.; Veenstra, T. D.; Udseth, H. R. An Accurate Mass Tag Strategy for Quantitative and High-Throughput Proteome Measurements. Proteomics 2002, 2, 513-523.

13. Burton, R. D.; Matuszak, K. P.; Watson, C. H.; Eyler, J. R. Exact Mass Measurements Using a 7 Tesla Fourier Transform Ion Cyclotron Resonance Mass Spectrometer in a Good Laboratory Practices-Regulated Environment. J. Am. Soc. Mass Spectrom. 1999, 10, 1291-1297.

14. Williams, D. K.; Muddiman, D. C. Parts-per-Billion Mass Measurement Accuracy Achieved Through the Combination of Multiple Linear Regression and Automatic Gain Control in a Fourier Transform Ion Cyclotron Resonance Mass Spectrometer. Anal. Chem. 2007, 79, 50585063.

15. Easterling, M. L.; Mize, T. H.; Amster, I. J. Routine Part-per-Million Mass Accuracy for High-Mass Ions: Space-Charge Effects in MALDI FT-ICR. Anal. Chem. 1999, 71, 624-632.

16. Muddiman, D. C.; Oberg, A. L. Statistical Evaluation of Internal and External Mass Calibration Laws Utilized in Fourier Transform Ion Cyclotron Resonance Mass Spectrometry. Anal. Chem. 2005, 77, 24062414.

17. Liu, T.; Belov, M. E.; Jaitly, N.; Qian, W. J.; Smith, R. D. Accurate Mass Measurements in Proteomics. Chem. Rev. 2007, 107, 3621-3653.

18. Zhang, L. K.; Rempel, D.; Pramanik, B. N.; Gross, M. L. Accurate Mass Measurements by Fourier Transform Mass Spectrometry. Mass Spectrom. Rev. 2005, 24, 286-309.

19. Francl, T. J.; Sherman, M. G.; Hunter, R. L.; Locke, M. J.; Bowers, W. D.; McIver, R. T. Experimental Determination of the Effects of Space Charge on Ion Cyclotron Resonance Frequencies. Int. J. Mass Spectrom. Ion Processes 1983, 54, 189-199.
20. Ledford, E. B.; Rempel, D. L.; Gross, M. L. Space-Charge Effects in Fourier-Transform Mass-Spectrometry-Mass Calibration. Anal. Chem. 1984, 56, 2744-2748.

21. Hannis, J. C.; Muddiman, D. C. A Dual Electrospray Ionization Source Combined with Hexapole Accumulation to Achieve High Mass Accuracy of Biopolymers in Fourier Transform Ion Cyclotron Resonance Mass Spectrometry. J. Am. Soc. Mass Spectrom. 2000, 11, 876-883.

22. O'Connor, P. B.; Costello, C. E. Internal Calibration on Adjacent Samples (InCAS) with Fourier Transform Mass Spectrometry. Anal. Chem. 2000, 72, 5881-5885.

23. Mize, T. H.; Amster, I. J. Broad-Band Ion Accumulation with an Internal Source MALDI-FTICR-MS. Anal. Chem. 2000, 72, 5886-5891.

24. Masselon, C.; Tolmachev, A. V.; Anderson, G. A.; Harkewicz, R.; Smith R. D. Mass Measurement Errors Caused by "Local" Frequency Perturbations in FTICR Mass Spectrometry. J. Am. Soc. Mass Spectrom. 2002, 13, 99-106.

25. Syka, J. E. P.; Marto, J. A.; Bai, D. L.; Horning, S.; Senko, M. W. Schwartz, J. C.; Ueberheide, B.; Garcia, B.; Busby, S.; Muratore, T.; Shabanowitz, J.; Hunt, D. F. Novel Linear Quadrupole Ion Trap/FT Mass Spectrometer: Performance Characterization and Use in the Comparative Analysis of Histone H3 Post-translational Modifications. J. Proteome Res. 2004, 3, 621-626.

26. Wong, R. L.; Amster, I. J. Sub Part-per-Million Mass Accuracy by Using Stepwise-External Calibration in Fourier Transform Ion Cyclotron Resonance Mass Spectrometry. J. Am. Soc. Mass Spectrom. 2006, 17, 16811691.

27. Wong, R. L.; Amster, I. J. Combining Low and High Mass Ion Accumulation for Enhancing Shotgun Proteome Analysis by Accurate Mass Measurement. J. Am. Soc. Mass Spectrom. 2006, 17, 205-212.

28. Marshall, A. G.; Wang, T. C. L.; Ricca, T. L. Tailored Excitation for Fourier Transform Ion Cyclotron Mass Spectrometry. J. Am. Chem. Soc. 1985, 107, 7893-7897.

29. Guan, S.; Marshall, A. G. Stored Waveform Inverse Fourier Transform (SWIFT) Ion Excitation in Trapped-Ion Mass Spectrometry: Theory and Applications. Int. J. Mass Spectrom. Ion Processes 1996, 157/158, 5-37.

30. Guan, S. General Phase Modulation Method for Stored Waveform Inverse Fourier Transform Excitation for Fourier Transform Ion Cyclotron Resonance Mass Spectrometry. J. Chem. Phys. 1989, 91, 775-777.

31. Marshall, A. G.; Guan, S. Advantages of High Magnetic Field for Fourier Transform Ion Cyclotron Resonance Mass Spectrometry. Rapid Commun. Mass Spectrom. 1996, 10, 1819-1823. 